\title{
Ensamblajes de peces arrecifales y su relación con el hábitat bentónico de la Isla Isabel, Nayarit, México
}

\author{
Reef fish assemblages and their relation with the benthic habitat of Isabel Island, Nayarit, México
}

\author{
Cristian M. Galván-Villa', José L. Arreola-Robles ${ }^{2}$, Eduardo \\ Ríos-Jara ${ }^{1}$ y Fabián A. Rodríguez-Zaragoza ${ }^{1}$
}

\begin{abstract}
${ }^{1}$ Laboratorio de Ecosistemas Marinos y Acuicultura, Departamento de Ecología, Centro Universitario de Ciencias Biológicas y Agropecuarias, Universidad de Guadalajara, Carretera a Nogales Km. 15,5, Las Agujas Nextipac, Zapopan CP 45110, Jalisco, México.edurios@cucba.udg.mx

${ }^{2}$ Instituto Tecnológico de Monterrey, Campus Guadalajara (ITESM-GDL), Av. General Ramón Corona \#2514, Col. Nuevo México, Zapopan CP 45201, Jalisco, México.
\end{abstract}

\begin{abstract}
This study describes the reef fish assemblages of Isabel Island and their relation with the benthos heterogeneity. A total of 39 visual transects from 19 different localities were performed in the zones North, South, East, and West around the island during December 2002, October 2004, and April 2005. The species composition and their abundance were registered in each transect. Video-transects were also performed to estimate the cover of benthic morpho-functional groups. A total of 9,956 specimens of fishes belonging to 118 species, 85 genera, and 51 families were registered. The $58.3 \%$ of the species have biogeographic affinity to the Panamic province. Six endemic species of the Mexican Pacific were registered, including two species endemic to the Gulf of California. According to the biological value index seven species were dominant in the island: Thalassoma lucasanum, Prionurus punctatus, Stegastes flavilatus, Halichoeres dispilus, Acanthurus xanthopterus, Abudefduf troschelii, and Johnrandallia nigrirostris. The indexes of diversity and evenness were not significantly different between the four zones of the island. However, there was a relation between the zones with higher biodiversity (South and East) and some components of the benthic habitat. The principal component analysis found that the cover of corals and rocks are highly related with the dominant species of the island, while the zones with highest proportion of sand, sponges, and gorgonians have less influence on fish diversity.
\end{abstract}

Key words: Ichthyofauna, diversity, abundance, biogeography, Gulf of California

Resumen.- En este estudio se describen los ensamblajes de peces arrecifales de la Isla Isabel y su relación con la heterogeneidad del bentos. Se realizaron 39 transectos visuales en 19 diferentes sitios de las zonas Norte, Sur, Este y Oeste alrededor de la isla durante los meses de diciembre de 2002, octubre de 2004 y abril de 2005. En cada transecto se registró la composición de especies y su abundancia. También se registraron video-transectos para estimar la cobertura de los grupos morfo-funcionales del bentos. Se registraron un total de 9.956 ejemplares de peces de 118 especies, 85 géneros y 51 familias. El 58,3\% de las especies presentan afinidad biogeográfica con la provincia Panámica. Se registraron seis especies endémicas del Pacífico mexicano, incluyendo dos endémicas del Golfo de California. De acuerdo con el índice de valor biológico, siete especies fueron dominantes en la isla: Thalassoma lucasanum, Prionurus punctatus, Stegastes flavilatus, Halichoeres dispilus, Acanthurus xanthopterus, Abudefduf troschelii y Johnrandallia nigrirostris. Los índices de diversidad y equidad no fueron significativamente diferentes entre las cuatro zonas de la isla. Sin embargo, se encontró una relación entre las zonas de mayor diversidad (Sur y Este) y algunos componentes del hábitat bentónico. El análisis de componentes principales encontró que las coberturas de coral y roca están altamente relacionas con las especies dominantes de la isla, mientras que las zonas con mayor proporción de arena, esponjas y gorgonáceos influyen en menor medida sobre la diversidad de peces.

Palabras clave: Ictiofauna, diversidad, abundancia, biogeografía, Golfo de California

\section{INTRODUCCIÓN}

Las formaciones arrecifales rocosas y coralinas del Pacífico mexicano albergan una gran diversidad ictiológica (Robinson \& Thomson 1992). A pesar de que la mayoría de los arrecifes de esta región son de tipo rocoso, los ensamblajes de peces de arrecifes coralinos han sido los más estudiados (e.g., Álvarez-Filip et al. 2006, Chávez-Comparan \& Macías-Zamora 2006, RamírezGutiérrez et al. 2007, Galván-Villa 2008), debido a que 
estos ecosistemas albergan una gran biodiversidad marina alcanzando un gran interés ecológico y turístico (Sale 1980, Reyes-Bonilla 1997).

El Golfo de California comprende abundantes islas con arrecifes rocosos en sus alrededores. La región presenta una variedad importante de peces ( $c a .875$ especies), comparable con la del Mar Rojo, el Golfo de México y las Bahamas (Thomson et al. 2000). Los ensamblajes de peces están compuestos por especies que se distribuyen tanto en la provincia de Cortez al norte como en la provincia Panámica al sur (Robertson \& Cramer 2009) e incluye la mayor cantidad de endemismos del Pacífico mexicano (Roberts et al. 2002).

La Isla Isabel se localiza al sureste de la boca del Golfo de California. La porción terrestre de la isla fue declarada Parque Nacional en 1980, dejando sin protección legal a la zona marina adyacente, la cual enfrenta intervenciones por actividades humanas como la pesca y el turismo (RíosJara et al. 2003).

Se han identificado algunos factores ambientales que influyen en la composición y abundancia de los peces asociados a arrecifes rocosos, principalmente la heterogeneidad estructural del hábitat (DominiciArosemena \& Wolff 2006) y la dinámica y exposición a las corrientes (Fulton et al. 2005). Estos mismos factores han sido señalados como importantes en los arrecifes rocosos de algunas islas e islotes del Golfo de California (AburtoOropeza \& Balart 2001, Villegas-Sánchez et al. 2009). Sin embargo, a pesar de la importancia de los ecosistemas marinos adyacentes a la isla y su gran diversidad biológica, en la Isla Isabel no se han realizado este tipo de estudios (Ríos-Jara et al. 2003, 2008) y el conocimiento sobre la ictiofauna marina de la isla es escaso, existiendo únicamente listados taxonómicos que refieren un total de 137 especies incluyendo peces óseos y cartilaginosos (Pérez-Jiménez 2001, Cano-Sánchez 2003, Ríos-Jara et al. 2008).

Por lo anterior, el objetivo principal del presente trabajo fue describir la estructura de los ensamblajes de peces asociados a los arrecifes rocosos de Isla Isabel y su relación con los rasgos fisiográficos y del bentos.

\section{Material Y MÉTOdos}

\section{Área de ESTUdio}

La Isla Isabel se encuentra aproximadamente a $28 \mathrm{~km}$ de la costa de Nayarit y $61,5 \mathrm{~km}$ al sureste de las Islas Marías $\left(21^{\circ} 50^{\prime} \mathrm{N}-105^{\circ} 53^{\prime} \mathrm{O}\right)$ (Fig. 1). Cuenta con una superficie total de 82,16 ha, su longitud máxima es de $1,8 \mathrm{~km}$ y su anchura promedio de $0,7 \mathrm{~km}$. La isla es de origen volcánico y presenta rasgos fisiográficos diferenciales que incluyen rocas expuestas con pendientes abruptas y altitudes variables, acantilados, pequeñas bahías, caletas y playas arenosas semi-protegidas al igual que playas rocosas con pendiente moderada y pozas de marea.

El área marina adyacente a la isla tiene una profundidad máxima de $36 \mathrm{~m}$ y pendientes suaves (1/200). En las zonas norte y este las pendientes varían desde planas hasta ligeramente onduladas, mientras que en las zonas oeste y sur llegan a ser moderadamente onduladas (Ríos-Jara et al. 2003). Alrededor de la isla sobresalen del lecho marino algunas formaciones rocosas, como "Las Monas" en la porción este y el "Cerro Pelón" en el noroeste de la isla. La disposición de la isla con respecto al viento, oleaje y las corrientes marinas dominantes, han originado una zona de mayor exposición en el norte y oeste de la isla. Esto se refleja en la configuración de su línea de costa ya que existe mayor acumulación de sedimentos en las bahías y caletas de las zonas Sur y Este, mientras que en las zonas del Norte y Oeste se presentan acantilados y playas rocosas.

\section{COMPOSICIÓN Y ABUNDANCIA}

Con base a las características fisiográficas y oceanográficas, el área de estudio se dividió en cuatro zonas: Norte, Sur, Este y Oeste, con un total de 19 localidades: seis en la zona Norte, cinco en la Sur, tres en la Este y cinco en la Oeste (Fig. 1). Los muestreos se realizaron en los meses de diciembre de 2002 (12 transectos), octubre de 2004 (17 transectos) y abril de 2005 (10 transectos). El efecto de la temporalidad se redujo realizando muestreos en cada una de las zonas de la isla en las dos principales temporadas del año: la temporada cálida (octubre y diciembre) y la temporada templada (abril). Se realizaron censos visuales mediante buceo SCUBA en transectos de 50 x $5 \mathrm{~m}$ y a profundidades entre 5 y $15 \mathrm{~m}$ (English et al. 1997). Cada censo fue realizado por un observador entre las 10:00 y 17:00 h, distribuidos de la siguiente forma: zonas Norte, Sur y Este con 11 transectos y zona Oeste con 6. En cada censo se registraron las especies de peces y sus abundancias (individuos $\mathrm{x}$ especie $\mathrm{x}$ transecto). Adicionalmente, se realizaron cuatro censos visuales y colectas para el registro de especies crípticas, en las localidades de Bahía Tiburoneros, Playa Iguanas, Las Monas y Cerro Pelón. Los especímenes recolectados fueron etiquetados y depositados en la colección de referencia de peces del 'Laboratorio de Ecosistemas Marinos y Acuicultura' de la Universidad de Guadalajara, México. Para la determinación taxonómica 


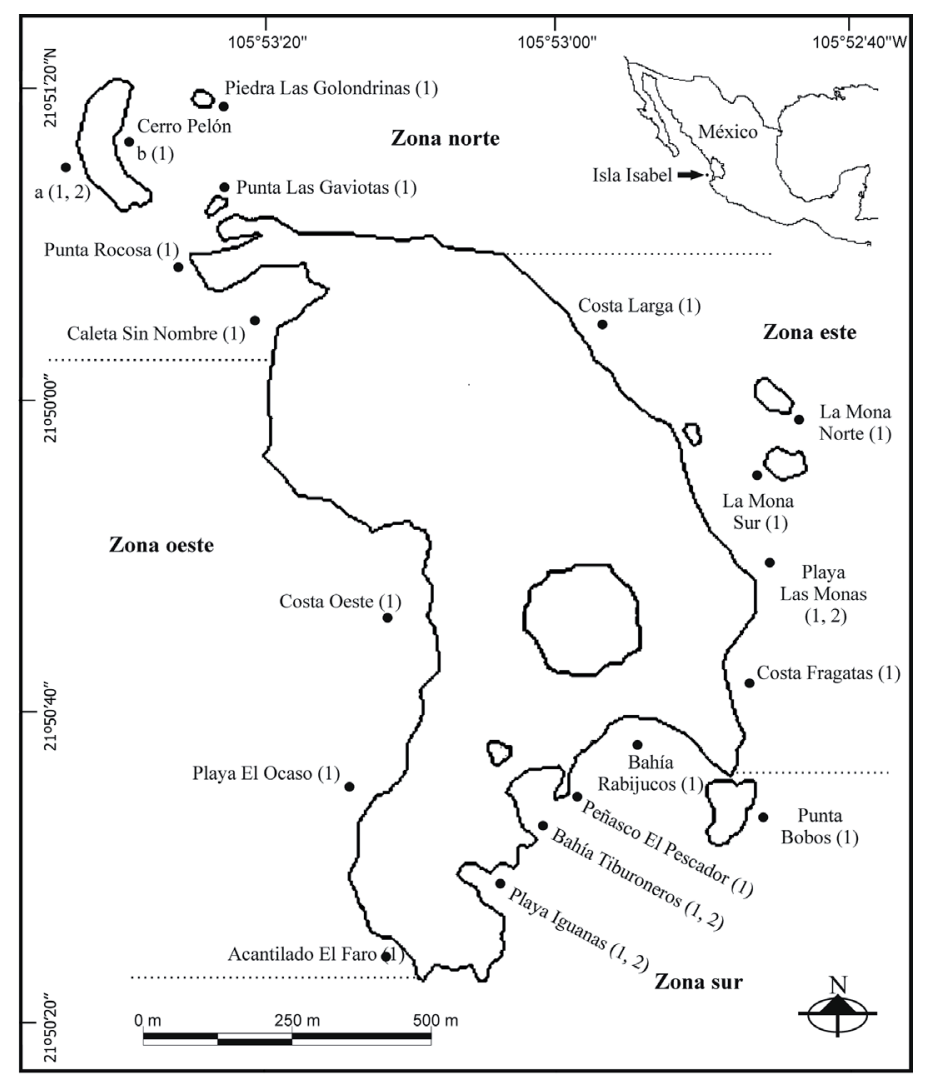

Figura 1. Localidades de muestreo en Isla Isabel (modificado de Ríos-Jara et al. 2003). (1) Sitios de conteos visuales, (2) sitios de recolectas con anestésico / Sampling localities at Isabel Island (modified from Ríos-Jara et al. 2003). (1) Sites of visual censuses, (2) sites of collection with anesthetics

de las especies se consultaron las guías de Kerstitch (1989), Allen \& Robertson (1994), Fischer et al. (1995), Thomson et al. (2000) y Humann \& Deloach (2004). El orden del listado sistemático se realizó según Nelson (2006) y las actualizaciones de Robertson \& Allen (2006).

El esfuerzo de muestreo ( $\mathrm{N}=39$ transectos $)$ fue evaluado con curvas de acumulación de especies (Mao Tau, Bootstrap, Chao1, Chao2 y Jackknife2). Las curvas se construyeron con 10.000 aleatorizaciones con el programa EstimateS v8.2 (Colwell 2006). Con los datos de riqueza específica, se analizó la afinidad zoogeográfica de las especies según los criterios de Allen \& Robertson (1994) y Hastings (2000).

\section{ESTRUCTURA COMUNITARIA}

La importancia de las especies en toda la isla y en cada una de las zonas Norte, Sur, Este y Oeste, se determinó mediante el Índice de Valor Biológico (IVB) (Sanders 1960), de acuerdo a lo propuesto por Loya-Salinas \& Escofet (1990). Para determinar la estructura del ensamblaje de peces, las especies fueron agrupadas en cinco categorías según los valores obtenidos por el IVB: especies dominantes (> 500 puntos), abundantes (201-500), comunes (101-200), escasas $(51-100)$ y raras $(\leq 50)$.

\section{ÍNDICES ECOLÓGICOS Y SIMILITUD DE ESPECIES}

La heterogeneidad de las especies de las cuatro zonas de la isla se analizó con los índices de diversidad de Shannon $\left(H^{\prime}\right)$, dominancia de Simpson $\left(D^{\prime}\right)$ y equidad de Pielou $\left(J^{\prime}\right)$ (Magurran 2004). Debido a la normalidad y homogeneidad de varianzas de los datos de los índices, se aplicó un análisis de varianza de una vía (ANDEVA) para saber si existen diferencias significativas entre las zonas (Zar 1999). 


\section{HeTERogeneidad del hábitAT BENTÓNICO Y RELACIÓN CON LOS ENSAMBLAJES DE PECES}

Se estimó la cobertura de diferentes elementos estructurales del hábitat en las cuatro zonas de la isla. Para ello se analizaron un total de 16 video-transectos de $50 \mathrm{~m}$ de longitud (cuatro por zona). La revisión de los videotransectos se realizó siguiendo la propuesta de AcostaGonzález (2005), que utiliza una serie de 13 puntos distribuidos sistemáticamente sobre el monitor y un total de 40 pausas para cada video-transecto. Se definieron cinco grandes grupos morfo-funcionales: roca, arena, esponjas, corales pétreos y gorgonáceos. La cobertura de cada grupo se estimó según la ecuación: $C=(\mathrm{Np} / 520)$ $x 100$, donde $C$ es el porcentaje de cobertura de cada grupo morfo-funcional y $N p$ es el número de puntos observados en las 40 imágenes de cada video-transecto (WWF 2006). La relación entre los grupos morfo-funcionales del hábitat y las zonas de la isla, así como entre las especies más importantes (dominantes y abundantes) y las zonas, se determinó mediante análisis de componentes principales (ACP). Los ACP se realizaron con PRIMER v6 (Clarke \& Gorley 2006) a partir de matrices construidas con la cobertura promedio por grupo morfo-funcional y la abundancia de las especies de peces más importantes del área de estudio.

\section{Resultados}

Se registró un total de 9.956 ejemplares de peces correspondientes a 118 especies, 84 géneros y 51 familias, de las cuales 100 especies se registraron mediante los censos visuales y 18 en las recolectas (Tabla 1). Las familias mejor representadas respecto al número de especies fueron Labridae (9), Pomacentridae (8), Labrisomidae (6), Lutjanidae (6), Muraenidae (5), Scaridae (5) y Serranidae (5). Las especies más abundantes fueron Thalassoma lucasanum (19,06\%), Prionurus punctatus (11,88\%) y Abudefduf troschelii $(11,75 \%)$ que en conjunto equivalen al $42,69 \%$ del total de peces censados.

Con el esfuerzo de muestreo realizado se logró un inventario de $\sim 72 \%$ de la riqueza específica estimada para la isla. El valor máximo de riqueza esperada (176 especies) fue con el estimador no paramétrico Chao2 (Fig. 2).

La ictiofauna de la Isla Isabel presenta una amplia distribución que incluye especies del Pacífico Oriental Tropical (66,6\%), Circuntropicales (16,7\%), de la región Pacífico Este (12,5\%) y del Indo-Pacífico (4,2\%). Las especies de la región del Pacífico Oriental Tropical presentan diferentes afinidades biogeográficas que incluyen a las provincias Panámica (58,3\%), del Mar de Cortés (4,2\%), Mexicana (3,3\%) y Californiana (0,8\%).

\section{ESTRUCTURA COMUNITARIA}

De acuerdo a los valores del IVB, siete especies resultaron dominantes en Isla Isabel: Thalassoma lucasanum, Prionurus punctatus, Stegastes flavilatus, Halichoeres dispilus, Acanthurus xanthopterus, Abudefduf troschelii y Johnrandallia nigrirostris. Estas especies mostraron abundancias relativas (AR) mayores al 3\% (máxima = $19,06 \%$ ) y porcentajes de ocurrencia sobre el $76 \%$, con excepción de $J$. nigrirostris que presentó una AR del $1,66 \%$ y una ocurrencia alta del $87,17 \%$. En conjunto representaron el 61,19\% de los peces censados (Fig. 3).

Las categorías de "abundantes", "comunes" y "escasas" incluyeron 14 especies cada una. Las primeras tuvieron valores de AR de entre 0,31 y 7,19\%, y frecuencias de ocurrencia entre 17,94 y 79,48\%; estas especies representaron en conjunto el $19,86 \%$ de todos los peces censados. Destacó Chromis atrilobata con la máxima $\operatorname{AR}(7,19 \%)$, pero con una frecuencia de ocurrencia baja (48,71\%). La AR de las especies "comunes" fue entre 0,15 y $3,76 \%$; en conjunto constituyen el $8,91 \%$ del total de peces censados con una frecuencia de ocurrencia de entre 7,69 y 48,71\%. La especies "escasas" presentaron valores de AR de entre 0,05 y $0,52 \%$, con excepción de Caranx sexfasciatus que obtuvo el valor máximo (3,24\%). Esta categoría representó el 5,46\% de los peces censados, con un rango de ocurrencia de entre 2,56 y 53,84\%. Finalmente, 51 especies fueron consideradas "raras" equivalentes al 4,55\% del total de peces. Los valores de AR fueron también bajos $(0,01-0,50 \%)$, con la excepción de Caranx caballus (2,08\%). Esta especie al igual que C. sexfasciatus, obtuvieron valores altos de AR en su respectiva categoría debido a que forman cardúmenes; aún así, la probabilidad de encontrarlas fue baja, por lo que sus porcentajes de ocurrencia fueron también bajos (C. sexfasciatus $=17,94 \%$, C. caballus $=5,12 \%$ ). Otras especies de esta última categoría presentaron un rango de ocurrencia amplio (2,56-46,15\%) (Fig. 3).

El orden de importancia de las especies según el IVB presentó variaciones entre las cuatro zonas (Fig. 4). Las seis especies más importantes (T. lucasanum, P. punctatus, S. flavilatus, $H$. dispilus, A. xanthopterus y A. troschelii) fueron constantes en toda la isla, aunque el orden de estas fue diferente entre cada zona. Además de las especies anteriores, en las zonas Norte y Este resalta la presencia de E. labriformis y H. nicholsi y en las zonas Sur y Oeste la presencia de $S$. verres. 


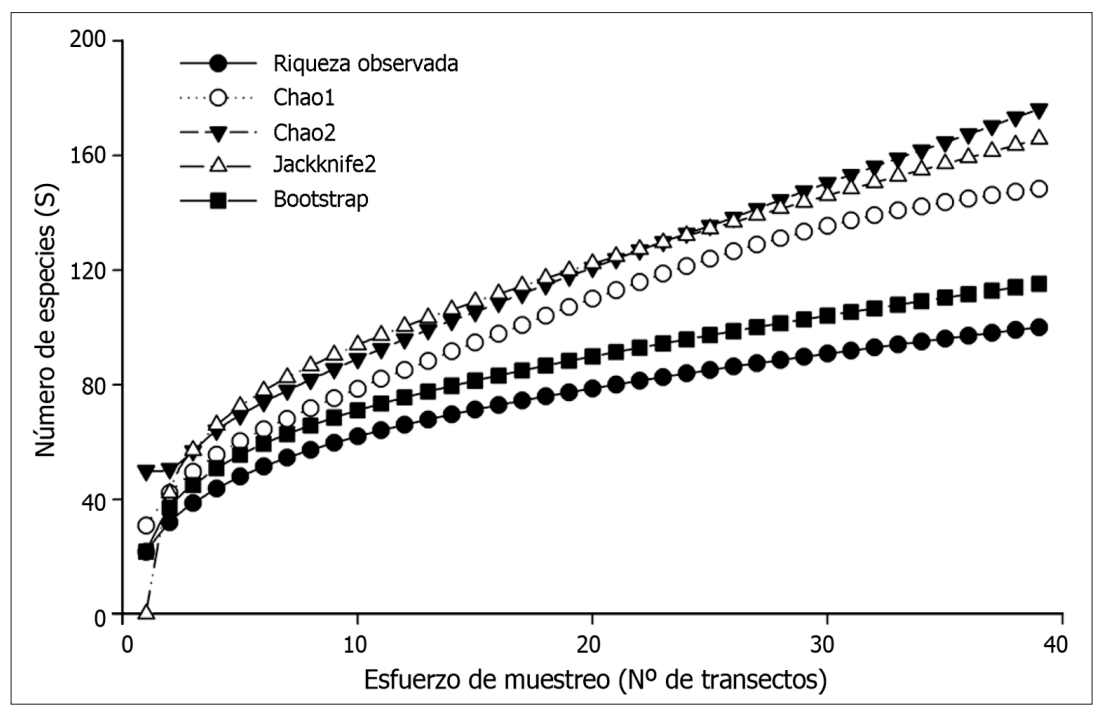

Figura 2. Curva de acumulación de especies de peces observadas (riqueza observada) y curvas de riqueza de especies estimadas con los estimadores no paramétricos Chao1, Chao2, Jackknife2 y Bootstrap / Observed fish species cumulative curve (observed richness) and estimated species richness curves calculated with non parametric estimators Chao1, Chao2, Jackknife2, and Bootstrap

\section{ÍNDICES ECOLÓGICOS Y SIMILITUD DE ESPECIES}

Los indicadores ecológicos utilizados permitieron reconocer zonas de mayor riqueza y diversidad de especies alrededor de la isla, así como de mayor dominancia y equidad (Tabla 2). En general, en las zonas Norte, Este y Oeste el número de especies fue de intermedio a bajo (4659 spp.), en comparación con la zona Sur (76 spp.). Los índices de diversidad, dominancia y equidad mostraron valores más bajos en las zonas Norte y Oeste $\left(H^{\prime} \leq 2,60 ; D^{\prime}\right.$ $\left.\leq 8,16 ; J^{\prime} \leq 0,64\right)$ que en las zonas Sur y Este $\left(H^{\prime} \geq 2,79 ; D^{\prime}\right.$ $\left.\geq 10,12 ; J^{\prime} \geq 0,68\right)$. Sin embargo, los análisis de varianza demostraron que no existen diferencias significativas entre las zonas para ninguno de los índices ecológicos $\left(H^{\prime}: \mathrm{F}=\right.$ $1,86, P=0,155 ; D^{\prime}: \mathrm{F}=1,50, P=0,232 ; J^{\prime}: \mathrm{F}=1,85, P$ $=0,155)$.

\section{HETEROGENEIDAD DEL HÁBITAT BENTÓNICO Y RELACIÓN CON LOS ENSAMBLAJES DE PECES}

La estructura del hábitat bentónico en cada una de las zonas presentó peculiaridades, a pesar de que el sustrato rocoso dominó en la mayor parte de la isla (83\%). En el análisis de componentes principales de la estructura béntica, los componentes 1 y 2 explicaron el $98,5 \%$ de la variación (Tabla 3), donde el primer componente separó a las zonas protegidas de la isla (Este y Sur) de las expuestas (Norte y Oeste) (Fig. 5a). En la zona Sur fueron importantes los sustratos coralinos, dominados por colonias del género
Pocillopora con una cobertura promedio en los videotransectos de 5,48\%. En la zona Este dominaron las formaciones rocosas $(87,59 \%)$ y colonias de coral dispersas $(2,93 \%)$. En contraste, las zonas Norte y Oeste presentaron mayor contribución de gorgonáceos (10,48 y 23,61\% respectivamente) y esponjas ( $0,04 \%$ en ambas zonas). El

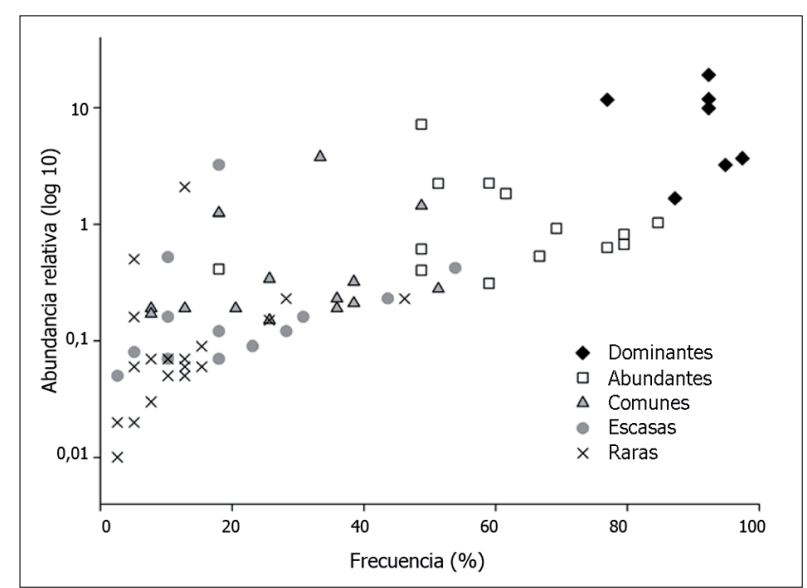

Figura 3. Estructura del ensamblaje de peces de Isla Isabel, en función de las categorías de importancia (dominantes, abundantes, comunes, escasas y raras) con respecto a su abundancia relativa (escala logarítmica) y al porcentaje de frecuencia de ocurrencia / Fish assemblage structure of Isabel Island based on categories of importance (dominant, abundant, common, scarce, and rare) with respect to their relative abundance (log scale) and frequency of occurrence percentage 
Tabla 1. Lista sistemática de los peces registrados en Isla Isabel, *especies recolectadas con anestésicos. Presencia en las diferentes zonas de la isla: $\mathbf{N}=$ Norte, $\mathrm{S}=$ Sur, $\mathrm{E}=$ Este, $\mathrm{O}=$ Oeste. Categorías de importancia: $\mathrm{D}=$ dominante, $\mathrm{A}=$ abundante, $\mathrm{C}=$ común, $\mathrm{E}=$ escasa, $\mathrm{R}=$ rara. Afinidad biogeográfica: $\mathrm{Ca}=$ Californiana, $\mathrm{Co}=$ Mar de Cortés, $\mathrm{Mx}=$ Mexicana, $\mathrm{Pa}=$ Panámica, $\mathrm{Pe}=\mathrm{Pac}$ fíco Este, Ip $=$ Indo-Pacífico, $\mathrm{Ct}=$ Circuntropical / Checklist of the fishes recorded at Isabel Island, *species collected with anesthetics. Presence in different zones of the island: $\mathrm{N}=$ North, $\mathrm{S}=$ South, $\mathrm{E}=$ East, $\mathrm{O}=$ West. Categories of importance: $\mathrm{D}=$ dominant, $\mathrm{A}=$ abundant, $\mathrm{C}=\mathrm{common}, \mathrm{E}=\mathrm{scarce}, \mathrm{R}=\mathrm{rare}$. Biogeographic affinity: $\mathrm{Ca}=$ Californian, $\mathrm{Co}=$ Mar de Cortés, $\mathrm{Mx}=$ Mexican, $\mathrm{Pa}=$ Panamic, $\mathrm{Pe}=$ Eastern Pacific, $\mathrm{Ip}=\mathrm{Indo}-\mathrm{Pacific}, \mathrm{Ct}=$ Circumtropical

\begin{tabular}{|c|c|c|c|c|c|c|c|c|c|}
\hline \multirow[t]{2}{*}{ Especie } & \multicolumn{4}{|c|}{ Zonas } & \multirow{2}{*}{$\begin{array}{l}\text { Abundancia } \\
\text { relativa } \\
(\mathrm{N}=9.956)\end{array}$} & \multirow{2}{*}{$\begin{array}{c}\text { Frecuencia de } \\
\text { ocurrencia } \\
\%\end{array}$} & \multirow{2}{*}{$\begin{array}{l}\text { Puntaje de } \\
\text { IVB }\end{array}$} & \multirow{2}{*}{$\begin{array}{l}\text { Categoría de } \\
\text { importancia }\end{array}$} & \multirow{2}{*}{$\begin{array}{c}\text { Afinidad } \\
\text { biogeográfica }\end{array}$} \\
\hline & $\mathrm{N}$ & $\mathrm{S}$ & $\mathrm{E}$ & $\mathrm{O}$ & & & & & \\
\hline \multicolumn{10}{|l|}{ Myliobatidae } \\
\hline Aetobatus narinari & $\mathrm{x}$ & & & & 0,03 & 7,69 & 1 & $\mathrm{R}$ & $\mathrm{Ct}$ \\
\hline \multicolumn{10}{|l|}{ Mobulidae } \\
\hline Manta birostris & & $\mathrm{x}$ & & & 0,01 & 2,56 & 1 & $\mathrm{R}$ & $\mathrm{Ct}$ \\
\hline \multicolumn{10}{|l|}{ Urolophidae } \\
\hline Urobatis halleri & $\mathrm{x}$ & & & & 0,07 & 10,25 & 1 & $\mathrm{R}$ & $\mathrm{Pe}$ \\
\hline \multicolumn{10}{|l|}{ Rhinobathidae } \\
\hline Zapteryx exasperata & & $\mathrm{x}$ & & & 0,02 & 5,12 & 1 & $\mathrm{R}$ & $\mathrm{Pe}$ \\
\hline \multicolumn{10}{|l|}{ Elopidae } \\
\hline Elops affinis & & $\mathrm{x}$ & & & 0,01 & 2,56 & 1 & $\mathrm{R}$ & $\mathrm{Pe}$ \\
\hline \multicolumn{10}{|l|}{ Muraenidae } \\
\hline Echidna nebulosa & $\mathrm{x}$ & & & & 0,01 & 2,56 & 1 & $\mathrm{R}$ & $\mathrm{Ct}$ \\
\hline Echidna nocturna* & & $\mathrm{x}$ & & & - & - & - & - & $\mathrm{Pe}$ \\
\hline Gymnomuraena zebra & $\mathrm{x}$ & & & & 0,01 & 2,56 & 1 & $\mathrm{R}$ & $\mathrm{Ct}$ \\
\hline Gymnothorax castaneus & $\mathrm{x}$ & $\mathrm{x}$ & $\mathrm{x}$ & & 0,07 & 17,94 & 56 & $\mathrm{E}$ & $\mathrm{Pa}$ \\
\hline Gymnothorax equatoralis* & & $\mathrm{x}$ & & & - & - & - & - & $\mathrm{Pa}$ \\
\hline Muraena lentiginosa & & $\mathrm{x}$ & & & 0,01 & 2,56 & 1 & $\mathrm{R}$ & $\mathrm{Pa}$ \\
\hline \multicolumn{10}{|l|}{ Chanidae } \\
\hline Chanos chanos & $\mathrm{x}$ & & & & 0,01 & 2,56 & 1 & $\mathrm{R}$ & $\mathrm{Ct}$ \\
\hline \multicolumn{10}{|l|}{ Synodontidae } \\
\hline Synodus evermanni* & & $\mathrm{x}$ & & & - & - & - & - & $\mathrm{Pa}$ \\
\hline Bythitidae & & & & & & & & & \\
\hline Ogilbia ventralis* & & $\mathrm{x}$ & & & - & - & - & - & $\mathrm{Pa}$ \\
\hline Batrachoididae & & & & & & & & & \\
\hline Porichthys margaritatus* & & $\mathrm{x}$ & & & - & - & - & - & $\mathrm{Pa}$ \\
\hline Antennaridae & & & & & & & & & \\
\hline Antennarius avalonis & & $\mathrm{x}$ & & & 0,01 & 2,56 & 1 & $\mathrm{R}$ & $\mathrm{Pa}$ \\
\hline Belonidae & & & & & & & & & \\
\hline Tylosurus pacificus & & & $\mathrm{x}$ & & 0,17 & 2,56 & 104 & $\mathrm{C}$ & $\mathrm{Ct}$ \\
\hline Holocentridae & & & & & & & & & \\
\hline Myripristies berndti & & $\mathrm{x}$ & & & 0,05 & 2,56 & 55 & $\mathrm{E}$ & $\mathrm{Ct}$ \\
\hline Myripristies leiognathus & $\mathrm{x}$ & $\mathrm{x}$ & & & 0,19 & 12,82 & 137 & $\mathrm{C}$ & $\mathrm{Pa}$ \\
\hline Sargocentrum suborbitalis & & $\mathrm{x}$ & $\mathrm{x}$ & & 0,06 & 12,82 & 17 & $\mathrm{R}$ & $\mathrm{Pa}$ \\
\hline Fistulariidae & & & & & & & & & \\
\hline Fistularia commersonii & $\mathrm{x}$ & $\mathrm{x}$ & $\mathrm{x}$ & $\mathrm{x}$ & 0,28 & 51,28 & 119 & $\mathrm{C}$ & $\mathrm{Pa}$ \\
\hline Scorpaenidae & & & & & & & & & \\
\hline Scorpaena mystes & & $\mathrm{x}$ & & & 0,01 & 2,56 & 1 & $\mathrm{R}$ & $\mathrm{Pa}$ \\
\hline Scorpaena russula* & & $\mathrm{x}$ & & & - & - & - & - & $\mathrm{Pa}$ \\
\hline Serranidae & & & & & & & & & \\
\hline Alphestes immaculatus & & $\mathrm{x}$ & & & 0,03 & 7,69 & 1 & $\mathrm{R}$ & $\mathrm{Pa}$ \\
\hline Cephalopholis panamensis & $\mathrm{x}$ & $\mathrm{x}$ & $\mathrm{x}$ & $\mathrm{x}$ & 0,40 & 48,71 & 301 & A & $\mathrm{Pa}$ \\
\hline Epinephelus labriformis & $\mathrm{x}$ & $\mathrm{x}$ & $\mathrm{x}$ & $\mathrm{x}$ & 0,82 & 79,48 & 480 & A & $\mathrm{Pa}$ \\
\hline Paranthias colonus & $\mathrm{x}$ & $\mathrm{x}$ & $\mathrm{x}$ & $\mathrm{x}$ & 0,19 & 20,51 & 161 & $\mathrm{C}$ & $\mathrm{Pa}$ \\
\hline Serranus psittacinus & & & $\mathrm{x}$ & & 0,02 & 5,12 & 1 & $\mathrm{R}$ & $\mathrm{Pa}$ \\
\hline Grammistidae & & & & & & & & & \\
\hline Rypticus bicolor & & $\mathrm{x}$ & $\mathrm{x}$ & & 0,02 & 5,12 & 29 & $\mathrm{R}$ & $\mathrm{Pa}$ \\
\hline Priacanthidae & & & & & & & & & \\
\hline Pristigenys serrula* & & $\mathrm{x}$ & & & - & - & - & - & $\mathrm{Pa}$ \\
\hline Apogonidae & & & & & & & & & \\
\hline Apogon pacifici & & $\mathrm{x}$ & & & 0,50 & 5,12 & 49 & $\mathrm{R}$ & $\mathrm{Pa}$ \\
\hline Apogon retrosella & $\mathrm{x}$ & $\mathrm{x}$ & & & 0,52 & 10,25 & 60 & $\mathrm{E}$ & $\mathrm{Pa}$ \\
\hline Echeneidae & & & & & & & & & \\
\hline Remora remora & $\mathrm{x}$ & & & & 0,02 & 2,56 & 1 & $\mathrm{R}$ & $\mathrm{Ct}$ \\
\hline Carangidae & & & & & & & & & \\
\hline Caranx caballus & $\mathrm{x}$ & & $\mathrm{x}$ & & 2,08 & 12,82 & 46 & $\mathrm{R}$ & $\mathrm{Pe}$ \\
\hline Caranx sexfasciatus & $\mathrm{x}$ & $\mathrm{x}$ & & $\mathrm{x}$ & 3,24 & 17,94 & 74 & $\mathrm{E}$ & $\mathrm{Ct}$ \\
\hline Seriola rivoliana & $\mathrm{x}$ & & & & 0,16 & 5,12 & 24 & $\mathrm{R}$ & $\mathrm{Ct}$ \\
\hline
\end{tabular}


Trachinotus rhodopus

Nematistiidae

Nematistius pectoralis

Gerreidae

Gerres cinereus

Lutjanidae

Hoplopagrus guntheri

Lutjanus argentiventris

Lutjanus guttatus

Lutjanus jordani

Lutjanus novemfasciatus Lutjanus viridis

Haemulidae

Anisostremus interruptus

Haemulon maculicauda

Haemulon sexfascitum

Haemulon steindachneri

Sparidae

Calamus brachysomus

Mullidae

Mulloidichthys dentatus

Kyphosidae

Kyphosus analogus

Kyphosus elegans

Ephippididae

Chaetodipterus zonatus

Chaetodontidae

Chaetodon humeralis

Johnrandallia nigrirostris

Pomacanthidae

Holacanthus passer

Pomacantus zonipectus

Pomacentridae

Abudefduf declivifrons

Abudefduf troschelii

Microspathodon bairdii

Microspathodon dorsalis

Stegastes acapulcoensis

Stegastes flavilatus

Stegastes rectifraenum

Chromis atrilobata

Cirrhitidae

Oxycirrhites typus

Cirrhitus rivulatus

Cirrhitichthys oxycephalus

Labridae

Bodianus diplotaenia

Halichoeres chierchiae

Halichoeres dispilus

Halichoeres melanotis

Halichoeres nicholsi

Halichoeres notospilus

Stethojulis bandanensis

Thalassoma grammaticum

Thalassoma lucasanum

Scaridae

Nicholsina denticulada

Scarus compresus

Scarus ghoban

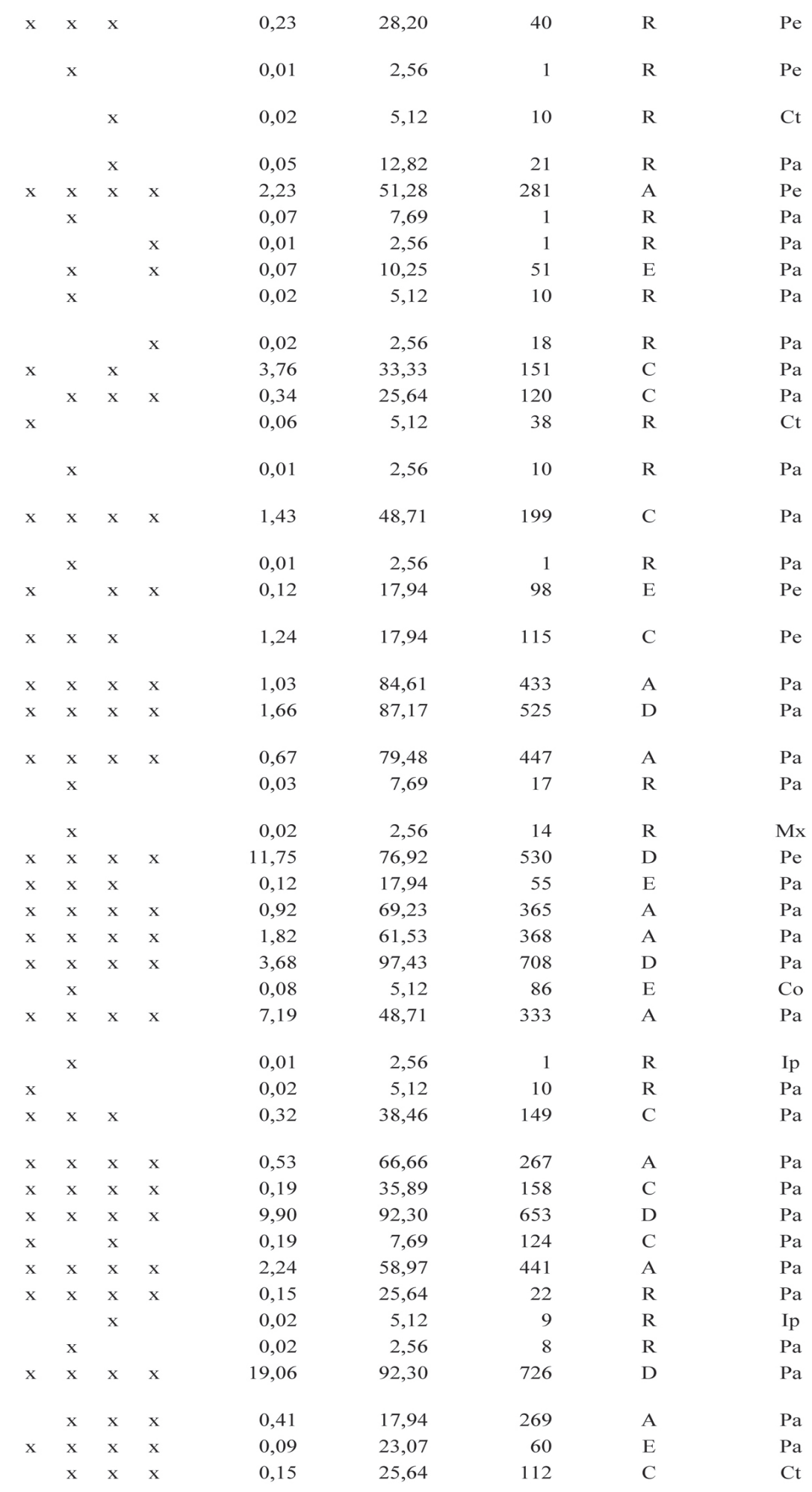

Vol. 45, N², 2010

317 


\begin{tabular}{|c|c|c|c|c|c|c|c|c|c|}
\hline Scarus perrico & $\mathrm{x}$ & $\mathrm{x}$ & $\mathrm{x}$ & $\mathrm{x}$ & 0,61 & 48,71 & 247 & A & $\mathrm{Pa}$ \\
\hline Scarus rubroviolaceus & $\mathrm{x}$ & $\mathrm{x}$ & $\mathrm{x}$ & & 0,23 & 43,58 & 64 & $\mathrm{E}$ & $\mathrm{Ct}$ \\
\hline \multicolumn{10}{|l|}{ Opistognathidae } \\
\hline Opistognathus rosenblatti & & $\mathrm{x}$ & & & 0,01 & 2,56 & 1 & $\mathrm{R}$ & Co \\
\hline \multicolumn{10}{|l|}{ Tripterygiidae } \\
\hline Crocodilichthys gracilis & & $\mathrm{x}$ & $\mathrm{x}$ & $\mathrm{x}$ & 0,12 & 28,20 & 67 & $\mathrm{E}$ & Co \\
\hline Enneanectes sexmaculatus* & & $\mathrm{x}$ & $\mathrm{x}$ & & - & - & - & - & $\mathrm{Pa}$ \\
\hline Enneanectes $s p{ }^{*}$ & & $\mathrm{x}$ & $\mathrm{x}$ & & - & - & - & - & $\mathrm{Pa}$ \\
\hline \multicolumn{10}{|l|}{ Dactyloscopidae } \\
\hline Gillellus searcheri* & & $\mathrm{x}$ & & & - & - & - & - & Co \\
\hline \multicolumn{10}{|l|}{ Labrisomidae } \\
\hline Malacoctenus ebisui* & & & $\mathrm{x}$ & & - & - & - & - & $\mathrm{Pa}$ \\
\hline Malacoctenus hubbsi* & & $\mathrm{x}$ & $\mathrm{x}$ & & - & - & - & - & $\mathrm{Mx}$ \\
\hline Malacoctenus margaritae & & & & $\mathrm{x}$ & 0,01 & 2,56 & 1 & $\mathrm{R}$ & $\mathrm{Pa}$ \\
\hline Malacoctenus zacae* & & & $\mathrm{x}$ & & - & - & - & - & $\mathrm{Mx}$ \\
\hline Paraclinus mexicanus* & & $\mathrm{x}$ & & & - & - & - & - & $\mathrm{Pa}$ \\
\hline Starksia spinipenis* & & $\mathrm{x}$ & & & - & - & - & - & $\mathrm{Mx}$ \\
\hline \multicolumn{10}{|l|}{ Chaenopsidae } \\
\hline Acanthemblemaria macrospilus & $\mathrm{x}$ & $\mathrm{x}$ & $\mathrm{x}$ & $\mathrm{x}$ & 0,05 & 12,82 & 19 & $\mathrm{R}$ & Co \\
\hline \multicolumn{10}{|l|}{ Bleniidae } \\
\hline Ophioblennius steindachneri & $\mathrm{x}$ & $\mathrm{x}$ & $\mathrm{x}$ & $\mathrm{x}$ & 0,16 & 30,76 & 97 & $\mathrm{E}$ & $\mathrm{Pa}$ \\
\hline Plagiotremus azaleus & $\mathrm{x}$ & $\mathrm{x}$ & $\mathrm{x}$ & $\mathrm{x}$ & 0,09 & 15,38 & 35 & $\mathrm{R}$ & $\mathrm{Pa}$ \\
\hline \multicolumn{10}{|l|}{ Gobidae } \\
\hline Bathygobius ramosus* & $\mathrm{x}$ & $\mathrm{x}$ & & $\mathrm{x}$ & - & - & - & - & $\mathrm{Pa}$ \\
\hline Coryphopterus urospilus* & & $\mathrm{x}$ & $\mathrm{x}$ & & - & - & - & - & $\mathrm{Pa}$ \\
\hline Elacatinus puncticulatus & $\mathrm{x}$ & & & & 0,01 & 2,56 & 2 & $\mathrm{R}$ & $\mathrm{Pa}$ \\
\hline \multicolumn{10}{|l|}{ Acanthuridae } \\
\hline Acanthurus nigricans & & $\mathrm{x}$ & & & 0,01 & 2,56 & 2 & $\mathrm{R}$ & $\mathrm{Pe}$ \\
\hline Acanthurus triostegus & $\mathrm{x}$ & $\mathrm{x}$ & $\mathrm{x}$ & & 0,16 & 10,25 & 59 & $\mathrm{E}$ & $\mathrm{Ct}$ \\
\hline Acanthurus xanthopterus & $\mathrm{x}$ & $\mathrm{x}$ & $\mathrm{x}$ & $\mathrm{x}$ & 3,24 & 94,87 & 619 & $\mathrm{D}$ & $\mathrm{Ct}$ \\
\hline Prionurus punctatus & $\mathrm{x}$ & $\mathrm{x}$ & $\mathrm{x}$ & $\mathrm{x}$ & 11,88 & 92,30 & 709 & $\mathrm{D}$ & $\mathrm{Pa}$ \\
\hline \multicolumn{10}{|l|}{ Zanclidae } \\
\hline Zanclus cornutus & $\mathrm{x}$ & $\mathrm{x}$ & $\mathrm{x}$ & & 0,21 & 38,46 & 131 & $\mathrm{C}$ & Ip \\
\hline \multicolumn{10}{|l|}{ Scombridae } \\
\hline Euthynnus lineatus & & $\mathrm{x}$ & & & 0,01 & 2,56 & 1 & $\mathrm{R}$ & $\mathrm{Pe}$ \\
\hline Scomberomorus sierra & & $\mathrm{x}$ & & & 0,02 & 2,56 & 21 & $\mathrm{R}$ & $\mathrm{Pe}$ \\
\hline \multicolumn{10}{|l|}{ Paralichthyidae } \\
\hline Cyclopsetta panamensis* & & $\mathrm{x}$ & & & - & - & - & - & $\mathrm{Pa}$ \\
\hline \multicolumn{10}{|l|}{ Balistidae } \\
\hline Balistes polylepis & $\mathrm{x}$ & $\mathrm{x}$ & & $\mathrm{x}$ & 0,07 & 12,82 & 44 & $\mathrm{R}$ & $\mathrm{Pe}$ \\
\hline Pseudobalistes naufragium & $\mathrm{x}$ & $\mathrm{x}$ & $\mathrm{x}$ & $\mathrm{x}$ & 0,23 & 46,15 & 45 & $\mathrm{R}$ & $\mathrm{Pa}$ \\
\hline Suflamen verres & $\mathrm{x}$ & $\mathrm{x}$ & $\mathrm{x}$ & $\mathrm{x}$ & 0,63 & 76,92 & 427 & $\mathrm{~A}$ & $\mathrm{~Pa}$ \\
\hline \multicolumn{10}{|l|}{ Monacanthidae } \\
\hline Aluterus scriptus & & & $\mathrm{x}$ & $\mathrm{x}$ & 0,05 & 10,25 & 38 & $\mathrm{R}$ & $\mathrm{Pe}$ \\
\hline \multicolumn{10}{|l|}{ Ostraciidae } \\
\hline Ostracion meleagris & $\mathrm{x}$ & & & $\mathrm{x}$ & 0,03 & 7,69 & 7 & $\mathrm{R}$ & $\mathrm{Ct}$ \\
\hline \multicolumn{10}{|l|}{ Tetraodontidae } \\
\hline Arothron hispidus & & & & $\mathrm{x}$ & 0,01 & 2,56 & 1 & $\mathrm{R}$ & Ip \\
\hline Arothron meleagris & $\mathrm{x}$ & $\mathrm{x}$ & $\mathrm{x}$ & $\mathrm{x}$ & 0,31 & 58,97 & 272 & A & Ip \\
\hline Canthigaster punctatissima & $\mathrm{x}$ & $\mathrm{x}$ & $\mathrm{x}$ & $\mathrm{x}$ & 0,42 & 53,84 & 82 & $\mathrm{E}$ & $\mathrm{Pa}$ \\
\hline \multicolumn{10}{|l|}{ Diodontidae } \\
\hline Diodon holocanthus & $\mathrm{x}$ & $\mathrm{x}$ & $\mathrm{x}$ & $\mathrm{x}$ & 0,23 & 35,89 & 192 & $\mathrm{C}$ & $\mathrm{Ct}$ \\
\hline Diodon hystrix & & $\mathrm{x}$ & $\mathrm{x}$ & $\mathrm{x}$ & 0,06 & 15,38 & 16 & $\mathrm{R}$ & $\mathrm{Ct}$ \\
\hline Especies censadas & 59 & 75 & 57 & 46 & & & & & \\
\hline Especies recolectadas & 1 & 16 & 6 & 2 & & & & & \\
\hline Total & 60 & 91 & 63 & 48 & & & & & \\
\hline
\end{tabular}


segundo componente principal, separó a las cuatro zonas con base en su cobertura de sustrato arenoso. Las zonas Norte y Este de la isla tuvieron porcentajes más altos de cobertura de arena $(11,11$ y $8,94 \%$ respectivamente) en comparación con las zonas Sur y Oeste (0,76 y 1,68\%).

El análisis de componentes principales del ensamblaje de peces mostró una asociación de las especies más importantes (dominantes y abundantes) en tres de las cuatro zonas de la isla (Fig. 5b). La variabilidad de los datos se explicó por el primer y segundo componente en más de un 79\% (Tabla 3). Las especies con mayor afinidad a la zona Este fueron: L. argentiventris, B. diplotaenia, $J$. nigrirostris, T. lucasanum y $C$. humeralis; las de la zona Norte: $S$. acapulcoensis, $H$. dispilus, $P$. punctatus, H. nicholsi, E. labriformis, A. troschelii, H. passer, S. flavilatus, A. xanthopterus y C. panamensis; y las de la zona Sur: $S$. verres, $N$. denticulata, $C$. atrilobata, $S$. perrico, M. dorsalis y A. meleagris (Fig. 5b).

La relación indirecta entre la estructura del hábitat bentónico y el ensamblaje de especies dominantes fue la siguiente: 1) En la zona Este, L. argentiventris, $B$. diplotaenia, J. nigrirostris, T. lucasanum y C. humeralis se correlacionan principalmente con un mayor porcentaje de sustrato rocoso y arenoso; 2) En la zona Norte, $S$. acapulcoensis, H. dispilus, P. punctatus, H. nicholsi, E. labriformis, A. troschelii, H. passer, S. flavilatus, A. xanthopterus y $C$. panamensis se relacionaron con coberturas más altas de arena y esponjas; 3) En la zona Sur, S. verres, N. denticulata, C. atrilobata, S. perrico, $M$. dorsalis y A. meleagris se asociaron con una mayor cobertura de corales y roca; y 4) En la zona Oeste, las densidades bajas de las especies se relacionaron a una mayor abundancia de gorgonáceos y esponjas (Fig. 5a, $5 b)$.

\section{Discusión}

El número de especies de peces registrado en la Isla Isabel (118) demuestra una riqueza superior a la reportada en otras islas del Golfo de California como Espíritu Santo con 80 especies (Arreola-Robles \& Elorduy-Garay 2002) y San José con 84 (Villegas-Sánchez et al. 2009). Esto adquiere mayor relevancia si se considera que la isla Isabel es relativamente pequeña $(<2 \mathrm{~km}$ de longitud $)$ en comparación con la mayoría de las islas del Golfo de

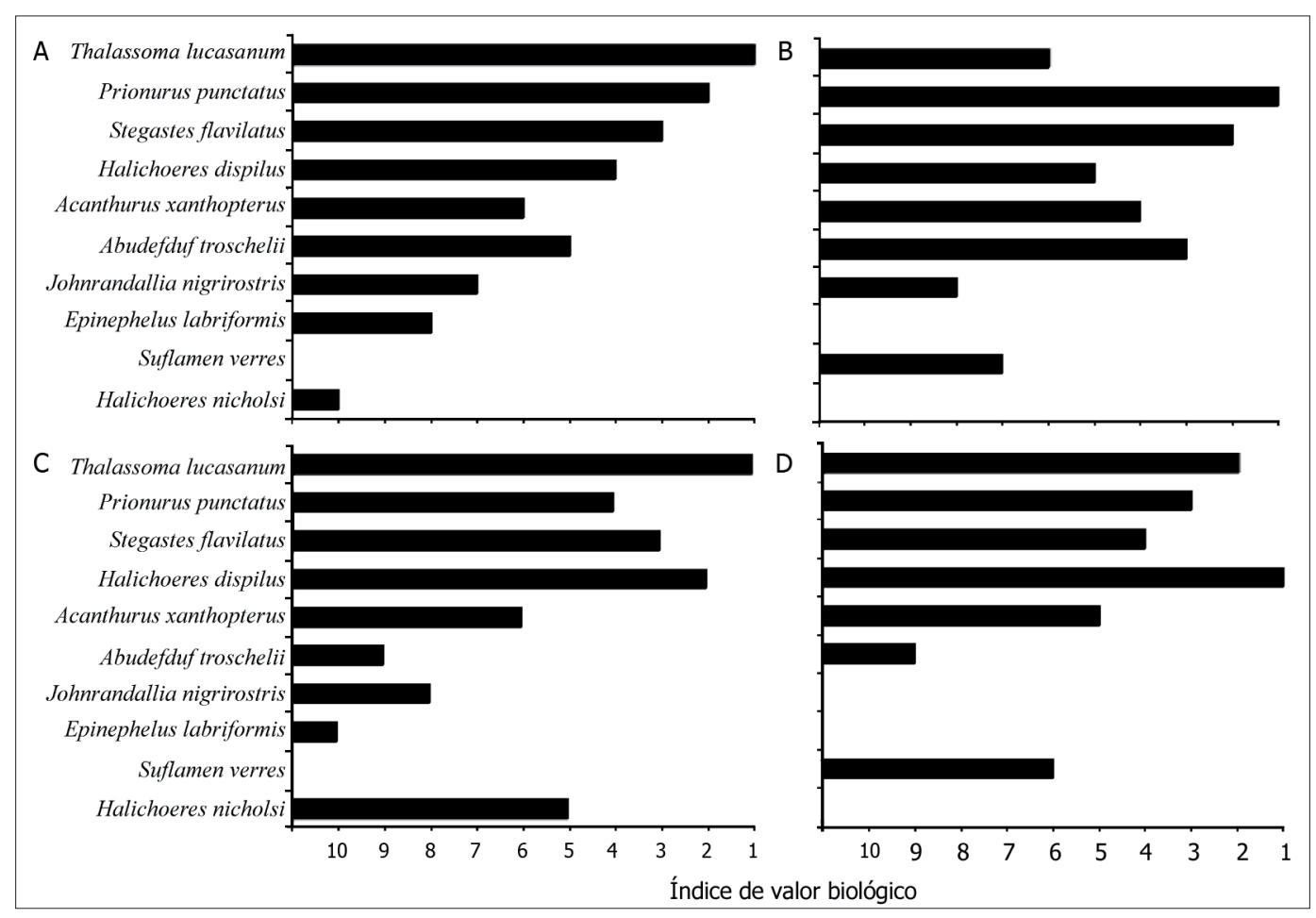

Figura 4. Posiciones de las especies dominantes dados por el Índice de Valor Biológico (IVB) durante los muestreos en las zonas Norte (A), Sur (B), Este (C) y Oeste (D) de Isla Isabel / Rank positions of dominant species according to the Biological Value Index (BVI) in the zones North (A), South (B), East (C), and West (D) of Isabel Island 
Tabla 2. Atributos comunitarios de los peces de Isla Isabel. Riqueza de especies $(S)$, diversidad $\left(H^{\prime}\right)$, dominancia $\left(D^{\prime}\right)$, equitatividad $\left(\mathrm{J}^{\prime}\right)$ y abundancia total $(\mathrm{ABT})$ de especies para cada zona de la isla; $\mathrm{gl}$ = grados de libertad, $\mathrm{P}=$ probabilidad de diferencia significativa en los valores de los índices ecológicos / Fish community attributes of Isabel Island. Species richness (S), diversity $\left(H^{\prime}\right)$, dominance $\left(D^{\prime}\right)$, evenness $\left(J^{\prime}\right)$, and total species abundance $(\mathrm{ABT})$ for each zone of the island; $\mathrm{gl}=$ degrees of freedom, $P=$ probability of significant difference in the ecological indexes value

\begin{tabular}{cccrccc}
\hline Índice / Zona & Norte & Sur & Este & Oeste & gl & $P$ \\
\hline$S$ & 59 & 75 & 57 & 46 & - & - \\
$H^{\prime}$ & 2,60 & 2,97 & 2,79 & 2,44 & 3 & 0,15 \\
$D^{\prime}$ & 8,16 & 11,64 & 10,12 & 7,47 & 3 & 0,23 \\
$J^{\prime}$ & 0,64 & 0,68 & 0,69 & 0,63 & 3 & 0,15 \\
$A B T$ & 3.223 & 2.395 & 2.876 & 1.462 & & \\
\hline
\end{tabular}

California (Rosabal 2005). Las diferencias en el número de especies estarían relacionadas con el esfuerzo y el tipo de muestreo. Cuando se complementan los censos visuales con diferentes métodos de colecta como redes agalleras, palangres, arpones y redes de mano, se puede incrementar notablemente el número de especies, como el estudio realizado por Galván-Magaña et al. (1996) en Isla Cerralvo que obtuvieron un registro de 174 especies.

Un componente importante en los arrecifes rocosos son las especies de hábitos crípticos que generalmente se subestiman en la mayoría de los inventarios de peces debido a las técnicas de muestreo utilizadas (Stewart $\&$ Beukers 2000). El uso de anestésicos para detectar especies crípticas permitió incrementar el inventario en la Isla Isabel ya que se registraron 18 especies crípticas adicionales que complementaron los registros de los censos visuales y que equivalen a un $16,8 \%$ de la riqueza específica total de la isla. Entre estas especies, cuatro son endémicas del Pacífico mexicano (Ogilbia ventralis, Acanthemblemaria macrospilus, Malacoctenus hubbsi y Opistognathus rosenblatti) y una del Golfo de California (Crocodilichthys gracilis), además de una especie no descrita del género Enneanectes. Esto es importante desde el punto de vista ecológico ya que el Golfo de California es reconocido como un centro de alto endemismo en el mundo (Roberts et al. 2002), con más de 800 especies de peces de las cuales entre un 25 y $30 \%$ son endémicas (CastroAguirre et al. 1995, Thomson et al. 2000). Es necesario intensificar el esfuerzo de captura, particularmente de las especies crípticas, además de ampliar la estacionalidad de los muestreos, para obtener el inventario esperado de 176 especies, dado por el estimador Chao2.
La estructura de la comunidad de peces se caracterizó por siete especies dominantes en toda la isla (T. lucasanum, P. punctatus, S. flavilatus, H. dispilus, A. xanthopterus, A. troschelii y $J$. nigrirostris), además de un gran número de especies escasas o raras las cuales se encuentran sólo en determinadas zonas (Tabla 1). El alto porcentaje de especies raras $(42,9 \%)$, dió como resultado una riqueza importante en la isla. A pesar de que la composición específica de peces fue distinta entre las zonas, los índices ecológicos de diversidad, dominancia y equidad fueron similares. Además, las especies dominantes mostraron una amplia distribución en la isla, por lo que es probable que la estructura del ensamblaje de peces sea constante a través del tiempo. Los cambios en la composición del ensamblaje de peces son comunes en la mayoría de los arrecifes rocosos y coralinos de la región sur del Golfo de California (Pérez-España et al. 1996, Arreola-Robles \& Elorduy-Garay 2002, Aburto-Oropeza \& Balart 2001, Álvarez-Filip et al. 2006). Usualmente estos cambios pueden estar relacionados con la variación estructural de los distintos hábitats del bentos; e.g., en la zona Norte, donde se encuentran paredes naturales que descienden hasta zonas arenosas, fueron frecuentes las especies de tallas medianas a grandes $(30-50 \mathrm{~cm})$ tales como $P$. punctatus, H. nicholsi, E. labriformis, H. passer, A. xanthopterus y $C$. panamensis; mientras que en la zona Este y Sur, caracterizadas por una amplia variedad de hábitats, se registraron especies de tallas medianas a pequeñas (10-30 $\mathrm{cm}$ ) como T. lucasanum, C. humeralis, J. nigrirostris, $B$. diplotaenia y L. argentiventris. Lo anterior, implica que las zonas más heterogéneas proveen de mejores espacios físicos para los peces de tamaños medianos y pequeños, ya que ofrecen mayores refugios de los depredadores y en

Tabla 3. Resultados del análisis de componentes principales. a) Relación entre cobertura de grupos morfofuncionales y zonas. b) Relación entre abundancia de especies importantes y zonas. $\mathrm{CP}=$ Componente Principal / Results of the principal component analysis. a) Relationship between of the morpho-functional groups cover and zones. b) Relationship between most important species abundance and zones. $\mathrm{CP}=$ Principal Component

\begin{tabular}{|c|c|c|c|c|}
\hline & $\mathrm{CP}$ & Autovalores & $\begin{array}{c}\text { Variación } \\
(\%)\end{array}$ & $\begin{array}{c}\text { Variación } \\
\text { acumulada (\%) }\end{array}$ \\
\hline \multirow[t]{2}{*}{ a) } & 1 & 3,4 & 67,9 & 67,9 \\
\hline & 2 & 1,53 & 30,5 & 98,5 \\
\hline \multirow[t]{2}{*}{ b) } & 1 & 9,22 & 43,9 & 43,9 \\
\hline & 2 & 7,4 & 35,2 & 79,2 \\
\hline
\end{tabular}




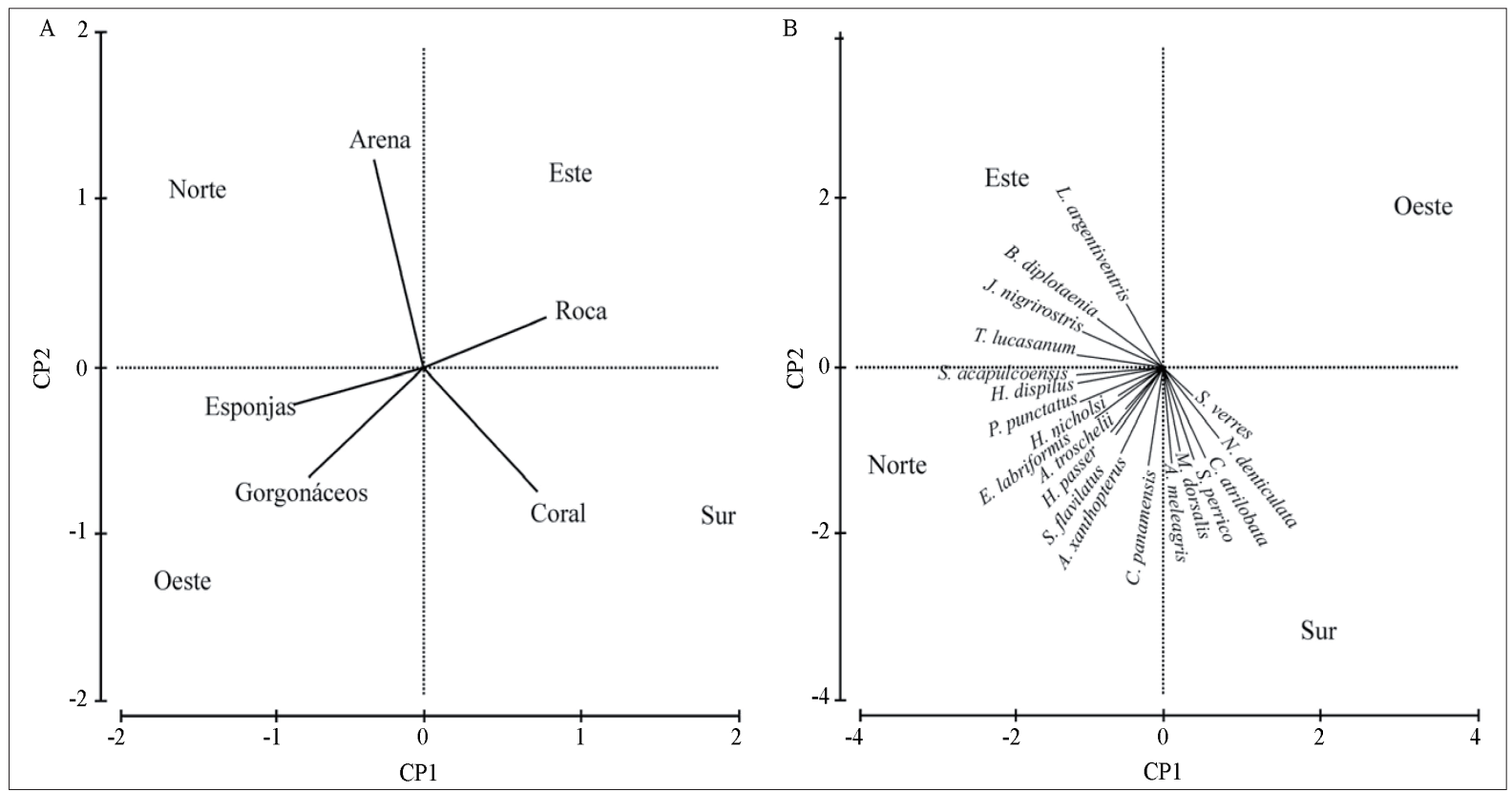

Figura 5. Análisis de componentes principales para relacionar la estructura del hábitat bentónico y el ensamblaje de peces en Isla Isabel. A. Ordenación de la cobertura de grupos morfo-funcionales del bentos y las zonas de la isla; B. Ordenación de la abundancia de especies importantes y las zonas. Los vectores corresponden a las contribuciones de las variables representativas del hábitat bentónico y de las especies de peces en cada zona de la isla / Principal components analysis to relate the benthic habitat structure and fish assemblages from Isabel Island. A. Ordination plot of benthic morpho-functional groups cover and island zones; B. Ordination plot of abundance of most important species and zones. Vectors correspond to representative variables contribution of benthic habitat and fish species at each zone of the island

ocasiones reducen la competencia (Jones 1988, Anderson et al. 1989).

En algunas islas costeras se ha observado que la composición de especies de peces varía considerablemente entre las zonas de alta y baja dinámica del oleaje (Depczynski \& Bellwood 2005, Fulton et al. 2005). Aunque en Isla Isabel esta condición no se presenta de forma significativa, los valores de dominancia de las especies tienden a aumentar en las zonas Sur y Este. Algunos ejemplos de sucesión de especies dominantes son Suflamen verres, la cual obtuvo los valores de importancia más altos en las zonas protegidas de la isla, encuentra refugio en las complejas estructuras rocosas y las colonias de coral de estas zonas; mientras que Chromis atrilobata, un pequeño pez planctófago que forma cardúmenes sobre los fondos rocosos, fue importante en las zonas expuestas de los arrecifes donde obtiene su alimento. Los patrones conductuales influyen también en la distribución y abundancia de las especies, por ejemplo Microspathodon dorsalis obtuvo su mayor abundancia en la zona Sur; esta es una especie solitaria y territorialista que prefiere los parches de arrecife rocoso donde deposita sus huevecillos en nidos que defiende con mayor agresividad durante la temporada reproductiva (Thomson et al. 2000).

La alta proporción de especies afines a las provincias Panámica y del Pacífico Este en la Isla Isabel coincide con Walker (1960), quien sugiere que la composición de especies del Golfo de California proviene principalmente de la región del Pacífico Oriental Tropical. Las islas del Océano Pacífico son consideradas puentes en la distribución de peces de diferentes regiones (Briggs 1961). Esto es particularmente importante en el caso de las especies que presentan larvas con largos periodos de permanencia en el plancton (Rosenblatt \& Waples 1986). Islas como Malpelo, Galápagos, Cocos, Clipperton y Revillagigedo, han permitido ampliar la distribución de especies del IndoPacífico hacia la costa oriental del Pacífico (Robertson et al. 2004). Ejemplos de tal situación en Isla Isabel son Oxycirrhites typus, Stethojulis bandanensis, Zanclus cornutus, Acanthurus nigricans, Acanthurus triostegus, Arothron meleagris, Arothron hispidus, entre otras, cuyo origen es el Indo-pacífico, incluso algunas de estas especies 
han sido reportadas en la costa continental de México, lo que sugiere que la Isla Isabel forma parte de este sistema insular que favorece la dispersión de las especies.

La información obtenida en el presente trabajo indicó que la distribución espacial de peces arrecifales alrededor de la Isla Isabel tiene relación con los elementos estructurales del bentos. Sin embargo, algunos patrones fueron difíciles de explicar debido a la escala espacial utilizada (zonas de la isla). Otros estudios han encontrado que las diferencias en la composición de los ensamblajes de peces arrecifales se deben en gran parte a la estructura del hábitat, especialmente a escalas pequeñas e intermedias (e.g., García-Charton et al. 2004). Es recomendable evaluar los ensamblajes de peces utilizando algunas otras características del hábitat como profundidad, número de oquedades en las rocas y complejidad topográfica, además de elementos morfo-funcionales más detallados como los tipos de estructuras coralinas y algales. Conocer detalladamente las relaciones entre los peces y el habitat ayudará de manera importante el desarrollo de mejores estrategias de manejo y conservación de los recursos marinos de la isla y de otros sistemas de arrecifes del Pacífico mexicano.

\section{Agradecimientos}

De manera especial queremos agradecer a Juan F. ElorduyGaray del CICIMAR-IPN y Felipe Amezcua del ICMyLUnidad Mazatlán, México por sus comentarios al trabajo. A Ross Robertson del Smithsonian Research Institute en Panamá, por su colaboración en la determinación de algunas especies. A Jorge Castrejón, Gonzalo Pérez y Cayetano Robles, personal del Parque Nacional Isla Isabel, por el apoyo durante el trabajo de campo. Finalmente, agradecemos a los revisores anónimos por sus observaciones y sugerencias al escrito, las cuales contribuyeron a mejorarlo. Este trabajo contiene información obtenida durante el proyecto BK018: "Inventario de la biota terrestre (florístico) y marina (invertebrados, peces y macroalgas bentónicos) del Parque Nacional Isla Isabel", financiado por la CONABIO, México.

\section{LITERATURA CITADA}

Aburto-Oropeza O \& EF Balart. 2001. Community structure of reef fish in several habitats of a rocky reef in the Gulf of California. Marine Ecology 22(4): 283-305.

Acosta-González G. 2005. Caracterización del hábitat arrecifal: hacia el desarrollo de SIGs en sistemas coralinos.
Tesis de Maestría, CINVESTAV-IPN, Unidad Mérida, Yucatán, 100 pp.

Allen GR \& DR Robertson. 1994. Fishes of the Tropical Eastern Pacific, 332 pp. University of Hawaii Press, Honolulu.

Álvarez-Filip L, H Reyes-Bonilla \& LE Calderón-Aguilera. 2006. Community structure of fishes in Cabo Pulmo Reef, Gulf of California. Marine Ecology 27: 253-262.

Anderson TW, EE De Martini \& DA Roberts. 1989. The relationship between habitat structure, body size and distribution of fishes in a temperate artificial reef. Bulletin of Marine Science 44: 681-697.

Arreola-Robles JL \& JF Elorduy-Garay. 2002. Reef fish diversity in the region of La Paz, Baja California Sur, Mexico. Bulletin of Marine Science 70(1): 1-18.

Briggs JC. 1961. The eastern Pacific barrier and the distribution of marine shore fishes. Evolution 15: 545-554.

Cano-Sánchez LE. 2003. Parque nacional Isla Isabel, 13 pp. Fichas Informativas de los Humedales de Ramsar (FIR), México.

Castro-Aguirre JL, EF Balart \& J Arvizu-Martínez. 1995. Contribución al conocimiento del origen y distribución de la ictiofauna del Golfo de California, México. Hidrobiológica 5(1-2): 57-78.

Chávez-Comparan JC \& R Macías-Zamora. 2006. Structure of reef fish communities in the littoral of Colima, Mexico. Journal of Biological Sciences 6(1): 65-75.

Clarke KR \& RN Gorley. 2006. PRIMER v6: User manual/ tutorial, 192 pp. PRIMER-E, Plymouth.

Colwell RK. 2006. EstimateS: Statistical estimation of species richness and share species from samples. Version 8.2. University of Connecticut, Storrs. [en línea] $<\mathrm{http} / / /$ viceroy.eeb.uconn.edu/estimates>

Depczynski M \& DR Bellwood. 2005. Wave energy and spatial variability in community structure of small cryptic coral reef fishes. Marine Ecology Progress Series 303: 283293.

Dominici-ArosemenaA\& M Wolff. 2006. Reeffish community structure in the Tropical Eastern Pacific (Panamá): living on a relatively stable rocky reef environment. Helgoland Marine Research 60(4): 287-305.

English S, C Wilkinson \& V Baker. 1997. Survey manual for tropical marine resources, $390 \mathrm{pp}$. Australian Institute of Marine Science, Townsville.

Fischer W, F Krupp, W Schneider, C Sommer, KE Carpenter \& VH Niem. 1995. Guía FAO para la identificación de especies para los fines de pesca. Pacífico Centro-Oriental. Vols. II y III. Vertebrados, partes 1 y 2, pp. 647-1813. FAO, Roma.

Fulton CJ, DR Bellwood \& PC Wainwright. 2005. Wave 
energy and swimming performance shape coral reef fish assemblages. Proceedings of the Royal Society B 272: 827832.

Galván-Magaña F, LA Abitia-Cárdenas, J RodríguezRomero, H Pérez-España \& H Chávez-Ramos. 1996. Lista sistemática de los peces de la Isla Cerralvo, Baja California Sur, México. Ciencias Marinas 22: 295-311.

Galván-Villa CM. 2008. Peces crípticos asociados a corales ramosos del género Pocillopora en el arrecife de Playa Mora, Bahía Tenacatita, México. Ciencia y Mar 11(34): 17-24.

García-Charton JA, A Pérez-Ruzafa, P Sánchez-Jerez, JT Bayle-Sempere, O Reñones \& D Moreno. 2004. Multiscale spatial heterogeneity, habitat structure, and the effect of marine reserves on Western Mediterranean rocky reef fish assemblages. Marine Biology 144: 161-182.

Hastings PA. 2000. Biogeography of the tropical eastern Pacific: Distribution and phylogeny of chaenopsid fishes. Zoological Journal of the Linnean Society 128: 319-335.

Humann P \& N Deloach. 2004. Reef fish identification, Baja to Panama, 343 pp. New World Publications, Jacksonville.

Jones GP. 1988. Experimental evaluation of the effects of habitat structure and competitive interactions on juveniles of two coral reef fishes. Journal of Experimental Marine Biology and Ecology 123: 115-126.

Kerstitch A. 1989. Sea of Cortez marine invertebrates: A guide for the Pacific coast, Mexico to Ecuador, 112 pp. Sea Challengers, Monterey.

Loya-Salinas DH \& A Escofet. 1990. Aportaciones al cálculo del Índice de Valor Biológico (Sanders 1960). Ciencias Marinas 16(2): 97-115.

Magurran AE. 2004. Measuring biological diversity, 256 pp. Blackwell Publishing, Malden.

Nelson JS. 2006. Fishes of the world, 601 pp. John Wiley \& Sons, New York.

Pérez-España H, F Galván-Magaña \& LAAbitia-Cárdenas. 1996. Variaciones temporales y espaciales en la estructura de la comunidad de peces de arrecifes rocosos del suroeste del Golfo de California, México. Ciencias Marinas 22(3): 273-294.

Pérez-Jiménez JC. 2001. Análisis de la pesquería artesanal de tiburones y rayas de Isla Isabel, Nayarit, México. Tesis de Maestría, Centro de Investigación Científica y de Educación Superior de Ensenada, Ensenada, 75 pp.

Ramírez-Gutiérrez M, M Tapia-García \& E RamosSantiago. 2007. Fish community structure in San Agustín Bay, Huatulco, Mexico. Revista Chilena de Historia Natural 80(4): 419-430.

Reyes-Bonilla H. 1997. Cabo Pulmo reef: a new marine reserve in the Gulf of California. Conservation Biology 11: 827.

Ríos-Jara E, M Pérez-Peña, E López-Uriarte, I EncisoPadilla, EG Robles-Jarero, A Martínez-Zataraín, $\mathbf{R}$ Maciel-Fores, V Davidova, JE Michel-Morfín \& CE López-Rivas. 2003. Estudio previo justificativo para la declaratoria bajo régimen de protección de la zona marina adyacente al Parque Nacional Isla Isabel, Informe interno, 154 pp. WWF-Programa Golfo de California, México.

Ríos-Jara E, E López-Uriarte, M Pérez-Peña, I EncisoPadilla, JL Arreola-Robles, A Hermosillo-González \& CM Galván-Villa. 2008. Listados taxonómicos de la biota marina del Parque Nacional Isla Isabel (invertebrados, peces y macroalgas), 194 pp. Universidad de Guadalajara, México.

Roberts CM, CJ McClean, JEN Veron, JP Hawkins, GR Allen, DE McAllister, CG Mittermeier, FW Schueler, M Spalding, F Wells, C Vynne \& TB Werner. 2002. Marine biodiversity hotspots and conservation priorities for tropical reefs. Science 295: 1280-1284.

Robertson DR \& GR Allen. 2006. Shorefishes of the tropical eastern Pacific: an information system. Smithsonian Tropical Research Institute, Balboa, Panamá. [CD-ROM].

Robertson DR \& KL Cramer. 2009. Shore fishes and biogeographic subdivisions of the Tropical Eastern Pacific. Marine Ecology Progress Series 380: 1-17.

Robertson DR, JS Grove \& JE McCosker. 2004. Tropical transpacific shore fishes. Pacific Science 58(4): 507-565.

Robinson J \& DA Thomson. 1992. Status of the Pulmo Coral Reefs in the lower Gulf of California. Environmental Conservation 19: 261-264.

Rosabal P. 2005. World heritage nomination IUCN summary: Islands and protected areas of the Gulf of California (Mexico), 54 pp. IUCN, Gland.

Rosenblatt RH \& RS Waples. 1986. A genetic comparison of allopatric populations of shore fish species from the eastern and central Pacific Ocean: dispersal or vicariance? Copeia 1986: 275-284.

Sale PF. 1980. The ecology of fishes on coral reefs. Oceanography and Marine Biology: an Annual Review 18: $367-421$.

Sanders HL. 1960. Benthic studies in Buzzard Bay. III. The structure of the soft-bottom community. Limnology and Oceanography 5: 138-153.

Stewart BD \& JS Beukers. 2000. Baited technique improves censuses of cryptic fish in complex habitats. Marine Ecology Progress Series 197: 259-272.

Thomson DA, LT Findley \& AN Kerstitch. 2000. Reef fishes of the Sea of Cortez. The rocky-shore fishes of the Gulf of California, 353 pp. The University of Texas Press, Austin. 
Villegas-Sánchez CA, LA Abitia-Cárdenas, FJ GutiérrezSánchez \& F Galván-Magaña. 2009. Rocky-reef fish assemblages at San José Island, Mexico. Revista Mexicana de Biodiversidad 80: 169-179.

Walker BN. 1960. The distribution and affinities of the marine fish fauna of the Gulf of California. Systematic Zoology 9(3): 123-133.
WWF. 2006. Mejores prácticas de pesca en arrecifes coralinos. Guía para la colecta de información que apoye el manejo de pesquerías basado en ecosistemas, 81 pp. WWF, México.

Zar JH. 1999. Biostatistical analysis, 672 pp. Prentice-Hall, Englewood Cliffs.

Recibido el 14 de agosto de 2009 y aceptado el 12 de mayo de 2010 